\title{
Middle Turbinate Mucosal Flap in Endoscopic Skull Base Reconstruction
}

\author{
Xuejian WANG, Xiaobiao ZHANG, Fan HU, Yong YU, Ye GU, Tao XIE, Junqi GE \\ Fudan University, Zhongshan Hospital, Department of Neurosurgery, Shanghai, China
}

\section{ABSTRACT}

AIM: To explore the indications, operation techniques and benefits of pedicled middle turbinate mucosal flap in endoscopic skull base reconstruction.

MATERIAL and METHODS: The patients, who underwent endonasal endoscopic surgery from October 2009 to June 2010 in our department, were involved in this study. Multi-layer skull base reconstruction was performed in four cases by pedicled middle turbinate mucosal flap combined with fascia lata, artificial dura mater, fat and other reconstruction materials. The results of surgery were assessed in the follow-up period.

RESULTS: No reconstruction-associated complications, such as cerebrospinal fluid leakage or infection were observed in four cases during the follow-up period for more than one year. All patients showed excellent recovery.

CONCLUSION: Pedicled middle turbinate mucosal flap for skull base reconstruction has the advantages of fast healing and low incidence of cerebrospinal fluid leakage. It provides satisfactory skull base reconstruction.

KEYWORDS: Reconstruction, Middle turbinate, Endoscope, Mucosal flap

\section{INTRODUCTION}

The application of neuroendoscopy in minimally invasive neurosurgery has been developing as a surgical method. At present, surgical indications of endonasal endoscopic surgery continue to expand from the front of anterior skull base to the odontoid and both sides of the middle skull base. Meanwhile, improvements in endoscopy technology have decreased surgery-related complications. Especially, cerebrospinal fluid (CSF) leakage, which is one of the postoperative complications related to endoscopy, is significantly reduced when nasal vascular pedicled mucosal flap combined with multi-layer reconstruction techniques is performed. The incidence of CSF leakage has been reported as less than $5 \%$ in the literature $(4,5)$. Endonasal endoscopic flaps such as pedicled nasal septum flap, pedicled middle turbinate flap and pedicled inferior turbinate flap are currently in use in surgical practice. The nasal septal mucosal flap is the most popular flap in endoscopic surgery. However, in some cases, pedicled nasal septum mucosal flap shows variations related to the substitutes of the pedicled mucosal flap.

Four cases were selected for pedicled middle turbinate mucosal flap and underwent endoscopic surgery between October 2009 and June 2010 in our department. The clinical results of these cases are presented.

\section{MATERIAL and METHODS}

\section{Clinical Data}

Four patients were male. The onset age of the symptoms in these patients was between 25 and 67 years, and the mean age was 45 years. The course of the symptoms was from one month to four years. The cases were 2 pituitary tumors, one craniopharyngioma and one spontaneous CSF leakage. The visual symptoms were the major clinical presentation. 
Decreased visual acuity was observed in three cases, disturbances in pituitary functions in two cases, the loss of olfaction in one case, headache in one case, and repeated intermittent nasal water flow in one case.

\section{Preoperative Treatment}

Based on preoperative magnetic resonance imaging (MRI) and computed tomography angiography (CTA), the location and size of the tumor and the anatomical structures of the nasal cavity were determined. Then, the indication of middle turbinate mucosal flap was discussed by the surgical team. Once the surgical decision was made, the patient was taken to the operating room.

\section{Surgical Method}

Nasal preparation: Following general anesthesia and oral intubation, the patient was in the supine position with the head slightly rotated to the right and fixed by three-nail head frame. After the routine disinfection procedure, the nasal cavity was infiltrated with $1 / 100000$ adrenaline and $0.5 \%$ lidocaine for 5 minutes to expand the operating space and to reduce bleeding.

Production of pedicled middle turbinate mucosal flap: The head of the middle turbinate was incised with unipolar electrocoagulation along the long axis (Figure 1A); both sides of the middle turbinate mucosa were stripped to the root of the middle turbinate along the periosteum (Figure 1B); the bare turbinate bone was blocked to bite by a rongeur the nasal cavity and the side wall of nasal cavity (Figure $1 \mathrm{C}$ ); the front edge of the middle turbinate was partly cut by scissors in order to attach to the cribriform plate (Figure 1D), and then the middle turbinate mucosa was formatted by two flap-like mucous membrane. One must be careful to not damage the cribriform plate during the operation as it may lead to iatrogenic CSF leakage. One must also pay special attention to protect the pedicle of the middle turbinate mucosal flap containing the middle turbinate branch of the sphenopalatine artery. In order to ensure the length and activity of the mucosal flap, the pedicle of the mucosal flap can be extended to the sphenopalatine foramen.
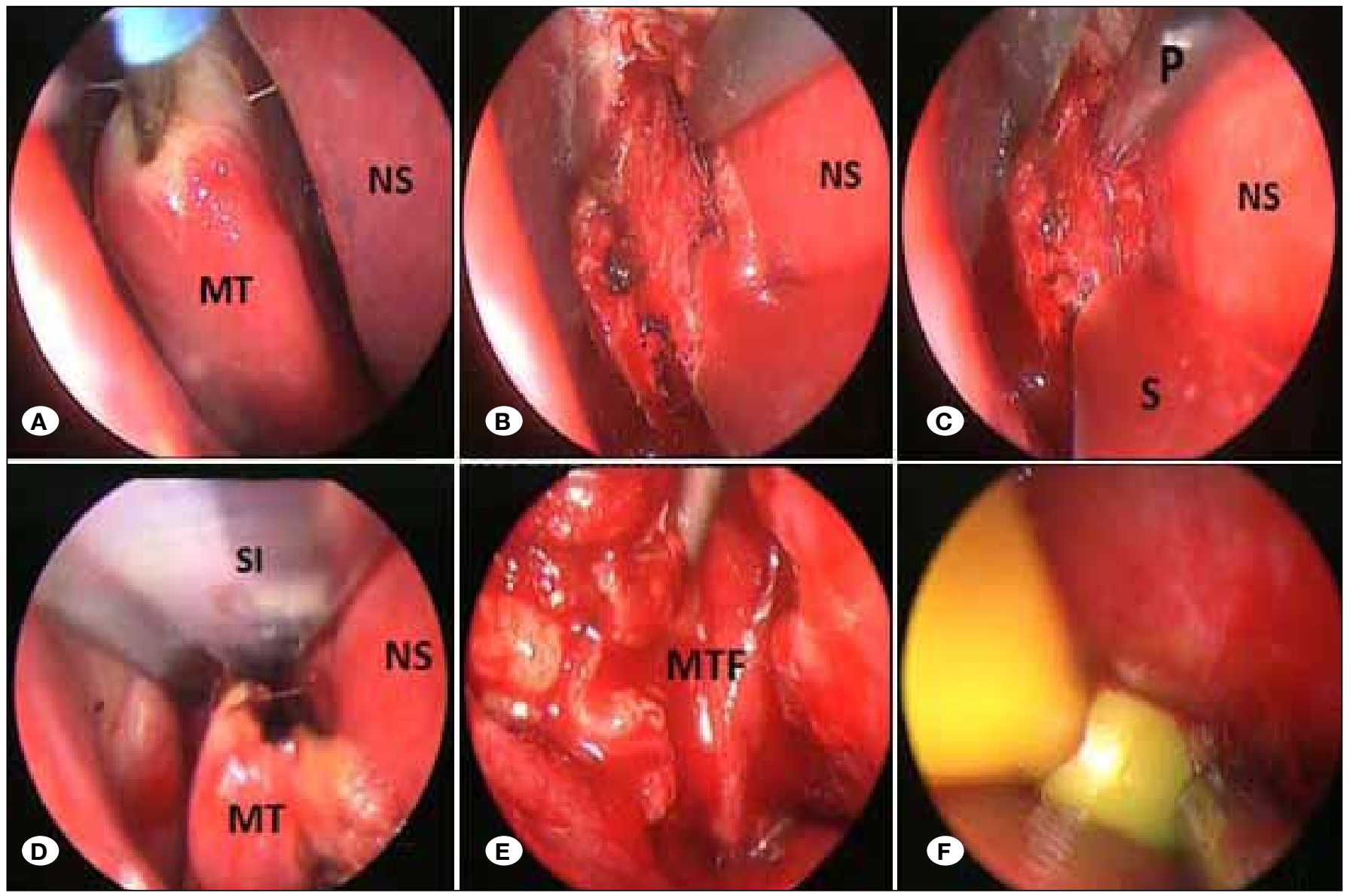

Figure 1: A) Straight incision of the middle turbinate along the long axis with monopolar cautery. B) Periosteal stripping of both sides of middle turbinate mucosa during the incision to the root of the middle turbinate. C) Block bite bare turbinate bone to the nasal cavity and skull base side wall by a rongeur. D) Cutting partially, by scissors, the front edge of the middle turbinate which is attached to the cribriform plate. E) Middle turbinate mucosal flap has been folded over the surgical wound at the end of surgery. F) Compression of the reconstruction by the $12^{\text {th }}$ Fogarty balloon catheter under endoscope. (NS: nasal septum; MT: middle turbinate; SI: scissors; P: rongeur; S: suction tube; MTF: middle turbinate mucosal flap). 
Multi-layer reconstruction of the skull base: For spontaneous CSF leakage case, the dural opening can be observed during the surgery. If the leakage is less than $0.5 \mathrm{ml}$, the intradural cavity should be filled with autobody, and the mucous membrane should be scrapped around the CSF leak site to form a fresh wound. The middle turbinate mucosal flap can be covered and fixed by biological glue. Finally, the mucosal flap was supported by the package of iodine spinning.

Artificial dura material was first used for intradural reconstruction; fascia lata and fat were used for epidural reconstruction in cases that underwent expanded transsphenoidal surgery. Then, the dural defect was covered by a middle turbinate mucosal flap (Figure 1E) and fixed by biological glue. Finally, the mucosal flap was supported by the $12^{\text {th }}$ Fogarty balloon catheter that was injected 8-10 ml water under endoscopic view (Figure 1F).

\section{Postoperative Management}

A lumbar puncture was performed for the continuous drainage of CSF in cases with intraoperative or postoperative CSF. Drainage volume depended on the patient, usually 100-200 $\mathrm{ml}$. per day. The drainage was generally maintained for 4-6 days, but this time may be extended when the CSF leakage continues or intracranial infection occurs. One week later, the nasal packing material was removed. Stool softening drugs should be administered if needed to keep the stool soft in these patients. In addition, these patients should avoid force or carrying heavy objects and coughing in the early postoperative period. All these conditions may increase the intracranial pressure leading to CSF leakage. Patients are followed-up regularly by examination after discharge, and the nose should be kept clean.

\section{- RESULTS}

Successful reconstruction was observed and CSF leakage, intracranial infection, pneumothorax or other complications were not detected in any patient. During the follow-up period of 1.25-2 years, the nasal mucosa revealed excellent growth in all cases without any complication.

\section{DISCUSSION}

Endonasal endoscopic technique in neurosurgery has developed rapidly and has now become an important part of minimally invasive neurosurgery. Thanks to the development of this technology, the incidence of surgery-related complications, such as CSF leakage and pneumocephalus, has significantly reduced. Carrabba et al. reported that the incidence of CSF leakage was $24 \%$ and tension pneumocephalus $6 \%$ after the extension of the endonasal transsphenoidal approach (1). Due to the improvement of reconstruction materials and reconstruction techniques, and especially pedicled autologous mucosal flaps to reconstruct the nasal cavity, the incidence of CSF leakage has reduced to less than $5 \%(4,7,15)$. Several kinds of nasal pedicled mucosal flaps including the nasal septum, middle turbinate and inferior turbinate mucosal flap have been applied. Among these, the pedicled nasal septum mucosal flap is the most popular, followed by the middle turbinate mucosal flap $(6,9)$.
The reasons of the use of a middle turbinate mucosal flap for the reconstruction of the skull base are as follows:

1) The location and extension of the surgical defect was evaluated and these were considered for reconstruction by middle turbinate mucosal flap.

2) In spontaneous CSF leakage case, the dural tear was in the sphenoethmoidal recess, which was close to the middle turbinate. Therefore, this flap was chosen for the reconstruction.

3) The middle turbinate mucosal flap was used as a supplementary to postoperative skull base reconstruction. The blood supply of the middle turbinate mucosal flap is located in the lateral wall of nasal cavity and is the branch of the lateral nasal artery, which should be protected during the surgery. This makes pedicled mucosal flap effective for reconstruction after surgery. However, the blood supply of the nasal septal mucosal flap is located in the channel of endoscopic surgery, and the mucosal flap cannot be protected and used if it is not prepared at the beginning of the surgery.

4) The pedicled middle turbinate mucosal flap is a good alternative when the nasal septum mucosa is damaged due to disease and other reasons.

5) Skilled endoscopic operative technique is required for the use of middle turbinate mucosal flap for the reconstruction. The surgeons in this study have the experience of more than 300 cases of endoscopic nasal surgery, and are familiar with the production of middle turbinate mucosal flap. The preparation of the middle turbinate mucosal flap is more difficult than a nasal septal mucosal flap. Especially, for the physician who is not familiar, the middle turbinate mucosal flap should not be chosen for reconstruction.

Although the use of a middle turbinate flap for the reconstruction has already been mentioned in the literature $(8,14)$, the pedicled middle turbinate mucosal flap is raised from a cadaver model by Prevedello et al. (11). Until 2011, Simal et al. have reported the satisfactory reconstruction with pedicled middle turbinate mucosal flap in a series of 10 cases that underwent the endonasal endoscopic approach (12). We do not advocate the pedicled middle turbinate mucosal flap as the first choice in surgery. First of all, the middle turbinate mucosal flap is relatively small. Prevedello et al. (11) and Simal et al. (12) confirmed that the effective area of the pedicled middle turbinate mucosal flap is 5-6 square centimeters. This is a very limited area and there is a need to fully assess and design in the preoperative period. At the same time, its operative requirements are relatively high. Based on our experience and reports in the literature, the nasal septum mucosa flap is relatively large, which can be enlarged up to 15 square centimeters for reconstructing the skull base defects in endonasal endoscopic surgery $(4,5)$. Second, the middle turbinate is attached to the side wall of the nasal cavity and improper operation may lead to iatrogenic CSF leakage and other complications. Third, the function of the middle turbinate is not clear. The middle turbinate can be moist, and has heating and antibody secretion functions. Nyquist et al. pointed out that although the middle 
turbinate function is still controversial, it should be kept intact during the surgery (10). In the process of producing a middle turbinate mucosal flap, its bone should be fully removed. Its anatomical structure cannot be restored after surgery and its functions are lost. In order to increase the operative space, the part of the middle turbinate should be resected during the production of nasal septal mucosal flap. In addition, in the postoperative period, the residual middle turbinate can still play a partial role in the healing of the mucous membranes of the nasal septum that can fully recover within the 2-3 months after the surgery.

The treatment by pedicled middle turbinate mucosal flap is usually combined with multi-layer reconstruction techniques. According to the literature and previous mucosal flap experience, we believed that even if the mucosal flap is successful, it still needs multi-layer reconstruction techniques. The mucosal flap is able to accelerate wound healing, and restore anatomical structures. However, multi-layer reconstruction is needed to obtain the support and fixation for reducing the influence of other factors, such as CSF soaking $(2,3,4,13)$. In our study, the pedicled middle turbinate mucosal flap was reconstructed with multi-layer reconstruction techniques, and postoperative follow-up examination was made for healing of mucous membranes in the surgical area. The anatomy and histology of the surgical site was also assessed in the follow-up examination in order to check the reconstruction of skull base.

Theoretically, the surgeon can choose any side of the middle turbinate in the production of mucosal flap. However, in practice, some factors such as hypertrophy, inflammation or deformity of the middle turbinate should be considered in the selection of mucosal flap. In our cases, we mostly choose the right side middle turbinate. However, in the spontaneous CSF leakage case, we chose the left side because the dural tear was in the left side.

There are some points that should be paid attention in the production and application of the mucosal flap process:

1. Nasal surgery needs to be done carefully and meticulously in order to reduce mucosal damage.

2. The incision of the mucosa must go in a straight line, and the edge of the wound should be neat.

3. The mucosal flap should not be damaged and the blood supply should be preserved during the procedure. Less burr application is required around the mucosal flap because burring may damage the mucosal flap

4. The periosteum of the mucosal flap must face the bone surface of the defect during the reconstruction process. The flattening and less folding of the mucosal flap are required in order to facilitate mucosal growth.

5. Balloon support plays an important role in ensuring the correct place of the mucosal flap and preventing the removal of the mucosal flap.
6. The placement of the balloon should be performed under the endoscope. This can ensure that the mucosal flap is in the right place and prevent the balloon mucosa oppression on the blood supply.

\section{CONCLUSION}

The pedicled middle turbinate mucosal flap to reconstruct skull base defects after transsphenoidal endoscopic surgery has multiple advantages such as fast healing and lower incidence of postoperative CSF leakage. At the same time, it enables excellent histological repair without rejection. Therefore, it is one of the important techniques for endoscopic endonasal reconstruction.

\section{- REFERENCES}

1. Carrabba G, Dehdashti AR, Gentili F: Surgery for clival lesions: Open resection versus the expanded endoscopic endonasal approach. Neurosurg Focus 25(6):E7, 2008

2. Cavallo LM, Messina A, Esposito F, de Divitiis O, Dal Fabbro M, de Divitiis E, Cappabianca P: Skull base reconstruction in the extended endoscopic transsphenoidal approach for suprasellar lesions. J Neurosurg 107(4): 713-720, 2007

3. El-Banhawy OA, Halaka AN, Altuwaijri MA, Ayad H, El-Sharnoby MM: Long-term outcome of endonasal endoscopic skull base reconstruction with nasal turbinate graft. Skull Base 18(5):297308, 2008

4. Hadad G, Bassagasteguy L, Carrau RL, Mataza JC, Kassam A, Snyderman $\mathrm{CH}$, Mintz $\mathrm{A}$ : A novel reconstructive technique after endoscopic expanded endonasal approaches: Vascular pedicle nasoseptal flap. Laryngoscope 116(10):1882-1886, 2006

5. Kassam A, Carrau RL, Snyderman CH, Gardner P, Mintz A: Evolution of reconstructive techniques following endoscopic expanded endonasal approaches. Neurosurg Focus 19(1):E8, 2005

6. Locatelli D, Rampa F, Acchiardi I, Bignami M, De Bernardi F, Castelnuovo P: Endoscopic endonasal approaches for repair of cerebrospinal fluid leaks: Nine-year experience. Neurosurgery 58(4) Suppl 2:ONS-246-256, 2006

7. Luginbuhl AJ, Campbell PG, Evans J, Rosen M: Endoscopic repair of high-flow cranial base defects using a bilayer button. Laryngoscope 120(5):876-880, 2010

8. Marks SC: Middle turbinate graft for repair of cerebral spinal fluid leaks. Am J Rhinol 12(6):417-419, 1998

9. Neligan PC, Mulholland S, Irish J, Gullane PJ, Boyd JB, Gentili F, Brown D, Freeman J: Flap selection in cranial base reconstruction. Plast Reconstr Surg 98(7):1159-1166, 1996

10. Nyquist GG, Anand VK, Brown S, Singh A, Tabaee A, Schwartz $\mathrm{TH}$ : Middle turbinate preservation in endoscopic transsphenoidal surgery of the anterior skull base. Skull Base 20(5): 343-347, 2010

11. Prevedello DM, Barges-Coll J, Fernandez-Miranda JC, Morera V, Jacobson D, Madhok R, dos Santos MC, Zanation A, Snyderman $\mathrm{CH}$, Gardner P, Kassam AB, Carrau R: Middle turbinate flap for skull base reconstruction: Cadaveric feasibility study. Laryngoscope 119(11):2094-2098, 2009 
12. Simal Julián JA, Miranda Lloret $P$, Cárdenas Ruiz-Valdepeñas E, Barges Coll J, Beltrán Giner A, Botella Asunción C: Middle turbinate vascularized flap for skull base reconstruction after an expanded endonasal approach. Acta Neurochir (Wien) 153(9):1827-1832, 2011

13. Tachibana E, Saito K, Fukuta K, Yoshida J: Evaluation of the healing process after dural reconstruction achieved using a free fascial graft. J Neurosurg 96(2):280-286, 2002
14. Xie Z, Jiang W, Zhang H, Zhao S, Xiao J: Transnasal endoscopic reconstruction of the nasal-skull base defect to manage the cerebrospinal fluid leaks with intranasal autologous grafts. Lin Chung Er Bi Yan Hou Tou Jing Wai Ke Za Zhi 22(17):769-771, 2008

15. Zanation AM, Carrau RL, Snyderman CH, Germanwala AV, Gardner PA, Prevedello DM, Kassam AB: Nasoseptal flap reconstruction of high flow intraoperative cerebral spinal fluid leaks during endoscopic skull base surgery. Am J Rhinol Allergy 23(5):518-521, 2009 